\title{
On Intersecting Chains in Boolean Algebras
}

\author{
PÉTER L. ERDŐS ${ }^{\dagger}$, ÁKOS SERESS ${ }^{\ddagger}$ and LÁSZLÓ A. SZÉKELY§ \\ ${ }^{\dagger}$ Centrum voor Wiskunde en Informatica, P.O. Box 4079, 1009 AB Amsterdam, The Netherlands \\ ¥The Ohio State University, Columbus, OH 43210 \\ $\S$ University of New Mexico, Albuquerque, NM 87131 \\ Received 15 April 1993; revised 18 October 1993
}

For Paul Erdős on his 80th birthday

\begin{abstract}
Analogues of the Erdős-Ko-Rado theorem are proved for the Boolean algebra of all subsets of $\{1, \ldots n\}$ and in this algebra truncated by the removal of the empty set and the whole set.
\end{abstract}

\section{Introduction}

One of the basic results in extremal set theory is the Erdős-Ko-Rado (EKR) Theorem [5]: if $\mathscr{F}$ is an intersecting family of $k$-element subsets of $[1, n]=\{1,2, \ldots, n\}$ (i.e. every two members of $\mathscr{F}$ have non-empty intersection) and $n \geq 2 k$, then $|\mathscr{F}| \leq\left(\begin{array}{l}n-1 \\ k-1\end{array}\right)$ and this bound is attained. We can consider $k$-subsets of $[1, n]$ as length- $k$ chains in the (total) order $1<2<\ldots<n$ : using this terminology, the EKR theorem is a result about intersecting $k$-chains in a special partially ordered set.

Erdős, Faigle, and Kern [3] pointed out that certain results of Deza, Frankl [2, Theorem 5.8], and Frankl and Füredi [7] on intersecting sequences of integers may be interpreted as results on intersecting families of chains in some partially ordered sets.

The purpose of this note is to prove analogues of the EKR theorem in two other partially ordered sets: in the Boolean algebra $\mathscr{B}_{n}$ of all subsets of $[1, n]$ (with $A \leq B$ if $A \subset B$ ), and in the truncated Boolean algebra $\mathscr{B}_{n}^{-}:=\mathscr{B}_{n} \backslash\{\emptyset,[1, n]\}$. We say that $\mathscr{L}=\left(L_{1}, L_{2}, \ldots, L_{k}\right)$ is a $k$-chain in $\mathscr{B}_{n}$ if $L_{i} \in \mathscr{B}_{n}$ for all $1 \leq i \leq k$ and $L_{i}$ is a proper subset of $L_{i+1}$ for all $1 \leq i \leq k-1$. A family $\mathscr{F}$ of $k$-chains in $\mathscr{B}_{n}$ is intersecting if any two elements of $\mathscr{F}$ have non-empty intersection.

$k$-chains and an intersecting family in $\mathscr{B}_{n}^{-}$are defined analogously. Let $f(n, k)$ and

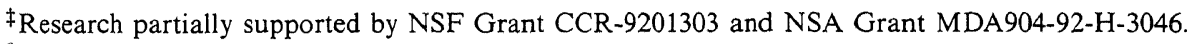

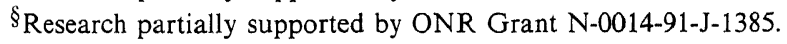


$f^{-}(n, k)$ denote the maximum size of intersecting families of $k$-chains in $\mathscr{B}_{n}$ and $\mathscr{B}_{n}^{-}$, respectively.

Obviously, the family $\mathscr{F}(A)$ of all $k$-chains containing some fixed $A \in \mathscr{B}_{n}$ is an intersecting family, and the same is true for the family $\mathscr{F}^{-}(A)$ of all $k$-chains in $\mathscr{B}_{n}^{-}$ containing some fixed $A \in \mathscr{B}_{n}^{-}$. Our main result is the following.

Theorem 1.1. For any $k, n$, we have

(i) $f(n, k)=|\mathscr{F}(\emptyset)|$ and

(ii) $f^{-}(n, k)=\left|\mathscr{F}^{-}(\{1\})\right|$.

Moreover, for $2 \leq k \leq n+1$, the only extremal families in $\mathscr{B}_{n}$ are $\mathscr{F}(\emptyset)$ and $\mathscr{F}([1, n])$.

The most well-known proof techniques for the original EKR Theorem are shifting and the kernel method. (For a brief introduction to these methods, see e.g., the survey papers of Frankl [6] and Füredi [8].) The kernel method usually ensures short and easy proofs, but rarely gives the exact range of the result. Shifting gives exact (but perhaps slightly more complicated) proofs.

The situation is very similar in our case: Z. Füredi (personal communication) showed, using only the kernel method, that for $n \geq 6 k \ln k$ Theorem 1.1(i) holds. In our proof of Theorem 1.1, we use an analogue of the shifting method and obtain a result without any restrictions on the parameters.

We remark, however, that to obtain sharp results in the case of $t$-intersecting families of chains, or the poset obtained by deleting the top $m$ and bottom $m$ levels in $\mathscr{B}_{n}$ for some $m<n / 2$, it seems to be necessary to combine the two methods. Hilton-Milner type generalizations are also possible. Moreover, we have a common generalization of the original EKR theorem and Theorem 1.1. We shall return to these problems in a forthcoming paper.

Let $S(p, q)$ denote the Stirling numbers of the second kind, i.e. $S(p, q)$ is the number of partitions of a $p$-element set into $q$ nonempty parts. It is easy to see that $|\mathscr{F}-(\{1\})|=$ $k ! S(n-1, k)$, since each $\mathscr{L}=\left(L_{1}, L_{2}, \ldots, L_{k}\right) \in \mathscr{F}-(\{1\})$ corresponds to an ordered partition $\left(L_{2} \backslash L_{1}, L_{3} \backslash L_{2}, \ldots, L_{k} \backslash L_{k-1},[1, n] \backslash L_{k}\right)$ of $[2, n]$. Similarly, $|\mathscr{F}(\emptyset)|=(k-1) ! S(n+1, k)=$ $(k-1) ! S(n, k-1)+k ! S(n, k)$, the two last terms corresponding to the number of $k$-chains in $\mathscr{F}(\emptyset)$ containing and not containing $[1, n]$, respectively.

In the proofs, we shall often use the well-known recursion

$$
S(n, k)=S(n-1, k-1)+k S(n-1, k)
$$

(see e.g., $[9$, Chapter 1]). In particular, $|\mathscr{F}(\emptyset)|=(k-1) ! S(n+1, k)$.

\section{Shifting}

In this section we begin the proof of Theorem 1.1. We reduce the problem to the examination of so-called compressed sets of chains and prove that these satisfy a strong intersection property.

Let $\mathscr{F}$ be a family of pairwise intersecting $k$-chains from $\mathscr{B}_{n}$ or $\mathscr{B}_{n}^{-}$, and let $1 \leq i<j \leq n$ be integers. The $(i, j)$ chain-shift $S_{i j}(\mathscr{F})$ of the family $\mathscr{F}$ is defined as follows. 
For every $k$-chain $\mathscr{L}=\left(L_{1}, \ldots L_{k}\right) \in \tilde{F}$, let $S_{i j}(\mathscr{L})=\left(L_{1}^{\prime}, \ldots, L_{k}^{\prime}\right)$, where

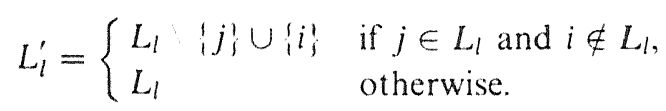

We say that $L_{j}^{\prime}$ is the shift of $L_{l}$. Shifting preserves set containment, so $S_{i j}(\mathscr{L})$ is a $k$-chain. The shifted family $S_{l j}(\mathscr{F})$ is obtained by the following rule: replace every $k$-chain $\mathscr{Z} \in \mathscr{F}$ by $S_{1 j}\left(\mathcal{X}^{\prime}\right)$ if and only if

(1) $S_{i j}\left(f^{\prime}\right) \neq \mathcal{L}^{\prime}$ and

(2) $S_{i j}(\mathscr{L}) \notin$.

It is clear from the definition that $\left|S_{i j}(\tilde{F})\right|=|\tilde{F}|$. Moreover, shifting preserves the intersection property.

Lemma 2.1. If $\mathscr{F}$ is an intersecting family of $k$-chains in $\mathscr{B}_{n}$ or $: \mathscr{B}_{n}^{-}$, then $S_{i j}(\tilde{F})$ is also intersecting.

Proof. Let $\mathscr{H}_{1}^{\prime}, \mathscr{f}_{2} \in S_{1 j}(\mathscr{F})$; we have to prove that they contain a common element. We distinguish three cases:

Case 1: $\mathscr{f}_{1}, \mathcal{L}_{2} \in \mathscr{F}$. In this case it is obvious that $\mathscr{f}_{1}$ and $\mathscr{f}_{2}$ intersect.

Case 2: $\mathscr{f}_{1}, \mathscr{f}_{2} \notin \mathscr{F}$. In this case, there are $\mathscr{L}_{3}, \mathscr{L}_{4} \in \mathscr{F}$ such that $\mathscr{H}_{1}=S_{i j}\left(\mathscr{L}_{3}\right)$ and $\mathscr{Y}_{2}=S_{1,}\left(\mathscr{Y}_{4}\right)$. Let $M \in \mathscr{L}_{3} \cap \mathscr{L}_{4}$. Then the shift of $M$ (which may be $M$ itself) is a common element of $f^{\prime} 1$ and $f_{2}^{\prime}$.

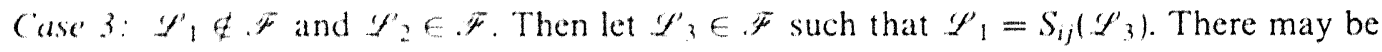
two reasons why $\mathscr{f}_{2}$ was not replaced. If $\mathscr{f}_{2}^{\prime}=S_{1 j}\left(\mathscr{f}_{2}\right)$ then let $M \in \mathscr{f}_{2} \cap \mathscr{f}_{3}$. The shift of $M$ is itself ( since $\mathscr{f}_{2}=S_{11}\left(\mathscr{f}_{2}\right)$ so $M \in \mathscr{f}_{2} \cap S_{1}\left(\mathscr{Y}_{3}\right)=\mathscr{f}_{2} \cap \mathscr{f}_{1}$ as well.

The other reason is that $\mathscr{f}_{2} \neq S_{1 j}\left(\mathscr{f}_{2}\right)$ but $S_{1 j}\left(\mathscr{H}_{2}\right) \in \mathscr{F}$. In this subcase, let $M \in$ $y_{3} \cap S_{11}\left(y_{2}\right)$ It is impossible that $j \in M$ and $i \notin M$ since $M$ is the shift of some element of $\mathscr{f}_{2}$. Also, it is impossible that $i \in M$ and $j \notin M$ because there is some $K \in \mathscr{P}_{3}$ such that $j \in K$ and $i \neq K$ (hecause $\left.S_{1}\left(\mathscr{Z}_{3}\right) \neq \mathscr{f}_{3}\right)$ and one of $K, M$ must contain the other. So $M$ is a set containing either hoth of $i, j$ or neither of $i, j$. In either case, from $M \in S_{i j}\left(\mathscr{L}_{2}\right)$ we have $M \in f^{\prime}$ so $M \in f_{1} \cap \mathcal{H}_{2}$.

We say that the family of intersecting $k$-chains is compressed if $\mathscr{F}$ is invariant for all chain-shift operations $S_{1}, 1 \leq i<j \leq n$. By Lemma 2.1, for any intersecting family $\mathscr{F}$, repeated applications of chain-shifts result in a compressed family of the same size.

Compressed families satisfy a strong intersection property. We say that $M \in \mathscr{B}_{n}$ for $\left.M \circ \mathscr{A}_{n}\right)$ is an initial segment if $M=[1, m]$ for some $1 \leq m \leq n$ or $M=\emptyset$.

I.emma 2.2. Let if be a compressed family of intersecting $k$-chains. Then for any $\mathscr{L}_{1}^{\prime}, \mathscr{L}_{2} \in$ F. F' and intersect in an initial segment.

Proof. Suppose that the lemma is not true and let $\mathscr{Y}_{1} \in \mathscr{F}$ be a minimal counterexample in the sense that

(i) there exists $\mathscr{f}_{2} \in \mathscr{F}$ such that $\mathscr{f}_{1} \cap \mathcal{L}_{2}$ contains no initial segment 
(ii) $\sum_{L \in \mathscr{L}_{1}} \sum_{x \in L} x$ is minimal among all $\mathscr{L}_{1}$ satisfying (i).

Let $M \in \mathscr{L}_{1} \cap \mathscr{L}_{2}$. Since $M$ is not an initial segment, there exist $1 \leq i<j \leq n$ such that $i \notin M$ and $j \in M$. Then $S_{i j}\left(\mathscr{L}_{1}\right) \neq \mathscr{L}_{1}$, so $S_{i j}\left(\mathscr{L}_{1}\right)$ is not a counterexample. Therefore, there exists an initial segment $K \in S_{i j}\left(\mathscr{L}_{1}\right) \cap \mathscr{L}_{2}$. It is impossible that $j \in K$ and $i \notin K$, since $K$ is an initial segment. Also, it is impossible that $i \in K$ and $j \notin K$, because $K, M \in \mathscr{L}_{2}$, so one of them must contain the other. So $K$ is a set containing both of $i, j$ or neither of $i, j$. In either case $K \in \mathscr{L}_{1}$, which is a contradiction.

In the next two sections, we prove Theorem 1.1 for $\mathscr{B}_{n}$ and $\mathscr{B}_{n}^{-}$, respectively. By Lemma 2.1, it is enough to consider compressed families.

\section{Chains in $\mathscr{B}_{n}$}

We prove by induction on $n$ that $f(n, k)=(k-1) ! S(n+1, k)$. The base case $n=1$ is trivial. Suppose we are done for $n-1$ (with all values of $k$ ) and let $\mathscr{F}$ be a compressed family of chains in $\mathscr{B}_{n}$. We distinguish two cases:

Case 1: $\mathscr{F}$ contains a chain $\mathscr{L}$ such that the only initial segment in $\mathscr{L}$ is $[1, n]$. Then, since each chain in $\mathscr{F}$ must intersect $\mathscr{L}$ in an initial segment (see Lemma 2.2), all chains contain $[1, n]$ and we are done.

Case 2: There is no chain in $\mathscr{F}$ such that $[1, n]$ is the only initial segment in the chain. Then delete $n$ from each element of each chain. Each chain is transformed into either a $k$-chain or a $(k-1)$-chain and so we obtain an intersecting family $\mathscr{C}_{k-1}$ of chains of length $k-1$ in $\mathscr{B}_{n-1}$ and an intersecting family $\mathscr{C}_{k}$ of chains of length $k$ in $\mathscr{B}_{n-1}$.

We claim that each $\left(L_{1}, \ldots, L_{k-1}\right) \in \mathscr{C}_{k-1}$ can be obtained from $\leq k-1$ chains of $\mathscr{F}$. This is true since we have to add the set $L_{i} \cup\{n\}$ to the chain for some $1 \leq i \leq k-1$ and add $n$ to the sets $L_{i+1}, \ldots, L_{k-1}$. The value of $i$ uniquely determines the chain in $\mathscr{F}$. Furthermore, $i=0$ is impossible, since then the only initial segment would be $[1, n]$.

We also claim that each $\left(L_{1}, \ldots, L_{k}\right) \in \mathscr{C}_{k}$ can be obtained from $\leq k$ chains of $\mathscr{F}$. Indeed, we have to add $n$ to the sets starting at some $2 \leq i \leq k+1$; the value $k+1$ corresponds to the case that $n$ did not occur in any element of the chain in $\mathscr{F}$. Furthermore, $i=1$ is impossible, since the only initial segment would be $[1, n]$.

Thus

$$
|\mathscr{F}| \leq(k-1) f(n-1, k-1)+k f(n-1, k)=(k-1) ! S(n+1, k) .
$$

The uniqueness of the extremal systems can also be proved by induction on $n$. First, we remark that if $k=n+1$, every family $\mathscr{F}$ of $k$-chains must contain the empty set, and maximality implies $\mathscr{F}=\mathscr{F}(\emptyset)$. If $k=2$ and $|\mathscr{F}| \geq 4$, then $\mathscr{F} \subseteq \mathscr{F}(A)$ for some subset $A$. Now, $|\mathscr{F}(A)|=2^{|A|}+2^{n-|A|}-2$, which takes its maximum value for $|A|=0$ and $|A|=n$.

In the case $3 \leq k \leq n$, we first consider a compressed family $\mathscr{F}$. If $\mathscr{F}$ belongs to Case 1 above, then $\mathscr{F}=\mathscr{F}([1, n])$; otherwise, in Case 2 , we must have equality in (1). This implies that $\mathscr{C}_{k-1}$ and $\mathscr{C}_{k}$ are extremal families in $\mathscr{B}_{n-1}$, and, by the induction hypothesis, they must be the $\mathscr{F}(\emptyset)$ of $(k-1)$-chains and $k$-chains in $\mathscr{B}_{n-1}$, respectively. So $\mathscr{F}$ must be identical with $\mathscr{F}(\emptyset)$ in $\mathscr{B}_{n}$. 
Finally, we observe that any family whose compressed image is $\mathscr{F}(\emptyset)$ or $\mathscr{F}([1, n])$ is itself one of these families.

We remark that in a preliminary version of the present paper [4], we proved Theorem 1.1 for $n>k \ln k$. Since then, Ahlswede and Cai [1] have also found a proof for Theorem 1.1 (i), but their method does not seem to generalize to the truncated case.

\section{Chains in $\mathscr{B}_{n}^{-}$}

Again, we use induction on $n$ to prove that $f^{-}(n, k)=k ! S(n-1, k)$. The base case $n=2$ is trivial. Suppose we are done for $n-1$ and let $\mathscr{F}$ be a compressed family of chains in $\mathscr{B}_{n}^{-}$. We distinguish two cases:

Case 1: If there exists a chain $\mathscr{L} \in \mathscr{F}$ such that $n-1 \in L_{1}$, then $\mathscr{L}$ may contain only one initial segment, namely $[1, n-1]$. Then, since each chain in $\mathscr{F}$ must intersect $\mathscr{L}$ in an initial segment (see Lemma 2.2), all chains contain $[1, n-1]$ and we are done.

Case 2: If each $\mathscr{L} \in \mathscr{F}$ has no $n-1 \notin L_{1}$, then, in particular, we never have $L_{1} \neq\{n-1\}$. Define

$$
\begin{aligned}
& \mathscr{F}_{i}=\left\{\mathscr{L} \in \mathscr{F}: L_{i+1}-L_{i}=\{n-1\}\right\}, \quad(i=1,2, \ldots, k-1) \\
& \mathscr{F}_{k}=\left\{\mathscr{L} \in \mathscr{F}: L_{k}=\{1,2, \ldots, n-2, n\}\right\}, \\
& \mathscr{F}_{0}=\mathscr{F}-\cup_{j=1}^{k} \mathscr{F}_{j} .
\end{aligned}
$$

Deleting $n-1$ from each element of each chain of $\mathscr{F}_{0}$, we obtain a family $\mathscr{F}_{0}^{\prime}$ of intersecting $k$-chains in the truncated Boolean algebra on the underlying set $\{1,2, \ldots, n-2, n\}$. By hypothesis, $\left|\mathscr{F}_{0}^{\prime}\right| \leq f^{-}(n-1, k)$. Each $\left(L_{1}, \ldots, L_{k}\right) \in \mathscr{F}_{0}^{\prime}$ can be obtained from $\leq k$ chains of $\mathscr{F}_{0}$, since $n-1$ could have been inserted starting at $L_{2}, L_{3}, \ldots, L_{k}$, or could have been an element of $[1, n] \backslash L_{k}$.

Deleting $n-1$ from every set in every chain in $\mathscr{F}_{i}$ (for any $i=1,2, \ldots, k-1$ ), we obtain a family $\mathscr{F}_{i}^{\prime}$ of intersecting $(k-1)$-chains in the truncated Boolean algebra on the underlying set $\{1,2, \ldots, n-2, n\}$. By hypothesis, $\left|\mathscr{F}_{i}\right|=\left|\mathscr{F}_{i}^{\prime}\right| \leq f^{-}(n-1, k-1)$.

Finally, define $\mathscr{F}_{k}^{\prime}$ by deleting the largest set $L_{k}=\{1,2, \ldots, n-2, n\}$ from every chain in $\mathscr{F}_{k}$. Observe that $\mathscr{F}_{k}^{\prime}$ is a family of intersecting $(k-1)$-chains in the truncated Boolean algebra on the underlying set $\{1,2, \ldots, n-2, n\}$, since the set that we dropped is not an initial segment in the original underlying set. Therefore, by hypothesis, $\left|\mathscr{F}_{k}\right|=\left|\mathscr{F}_{k}^{\prime}\right| \leq$ $f^{-}(n-1, k-1)$.

Hence, $|\mathscr{F}| \leq k \cdot k ! S(n-2, k)+(k-1)(k-1) ! S(n-2, k-1)+(k-1) ! S(n-2, k-1)=$ $k ! S(n-1, k)$.

This finishes the proof of Theorem 1.1.

We remark that, analogously to the discussion at the end of Section 3, it can be shown that the only compressed extremal families in $\mathscr{B}_{n}^{-}$are $\mathscr{F}^{-}([1])$ and $\mathscr{F}^{-}([1, n-1])$. The extension that the only extremal families are $\mathscr{F}^{-}(A)$ with $|A|=1$ or $|A|=n-1$ is still missing. 


\section{Acknowledgements}

We are indebted to Ulrich Faigle, Zoltán Füredi, and Walter Kern for stimulating conversations on the subject of the paper.

\section{Note added in proof}

We have just learned of a research program initiated by Miklós Simonovits and Vera T. Sós on 'structured intersection theorems' $[10,11]$, which has a fairly large literature. They studied the maximum number of graphs on $n$ vertices such that any two intersect in a prescribed graph, e.g. a path or cycle. The following problem fits into their scheme: given a graph $G$ what is the maximum number of pairwise intersecting complete $k$-subgraphs. In this paper we have studied the comparison graphs of some partially ordered sets.

\section{References}

[1] Ahlswede, R. and Cai, N. (1993) Incomparability and intersection properties of Boolean interval lattices and chain posets, preprint.

[2] Deza, M. and Frankl, P. (1983) Erdős-Ko-Rado theorem - 22 years later, SIAM J. Alg. Disc. Methods 4, 419-431.

[3] Erdős, P. L., Faigle, U. and Kern, W. (1992) A group-theoretic setting for some intersecting Sperner families, Combinatorics, Probability and Computing 1, 323-334.

[4] Erdős, P. L., Seress, Á. and Székely, L. A. (1993) On intersecting k-chains in Boolean algebras, Preprint, April 1993.

[5] Erdős, P., Ko, C. and Rado, R. (1961) Intersection theorems for systems of finite sets, Quart. J. Math. Oxford Ser. 2 12, 313-318.

[6] Frankl, P. (1987) The shifting technique in extremal set theory, In: Whitehead, C. (ed.) Surveys in Combinatorics 1987, Cambridge University Press, 81-110.

[7] Frankl, P. and Füredi, Z. (1980) The Erdős-Ko-Rado theorem for integer sequences, SIAM J. Alg. Disc. Methods 1, 376-381.

[8] Füredi, Z. (1991) Turán type problems, In: Keedwell, A. D. (ed.) Surveys in Combinatorics 1991, Cambridge University Press 253-300.

[9] Lovász, L. (1977) Combinatorial Problems and Exercises, Akadémiai Kiadó, Budapest and North-Holland, Amsterdam.

[10] Simonovits, M. and Sós, V. T., Intersection theorems for graphs. Problèmes Combinatoires et Theorie des Graphes, Coll. Internationaux C.N.R.S. 260 389-391.

[11] Simonovits, M. and Sós, V. T. (1978) Intersection theorems for graphs II. Combinatorics, Coll. Math. Soc. J. Bolyai 18 1017-1030. 